\title{
NEW STANDARDS FOR THE SPECTRAL \\ CLASSIFICATION OF M DWARFS
}

\author{
R. F. WING \\ Perkins Observatory, The Ohio State and Ohio Wesleyan Universities
}

\begin{abstract}
Spectral types based upon photoelectric measurements of the strong TiO band near $7100 \AA$ and having an internal accuracy of one-tenth of a subclass are presented for 26 dwarf stars in the range $\mathrm{K} 4$ to M6. For the calibration of the TiO index into spectral type, the MK scale for giants established by Wing and Keenan was adopted. Accordingly the types differ systematically from those previously published on any of the systems used for dwarfs, being closest to Joy's. The advantages of the MK giant scale are discussed, and it is suggested that the stars classified here be adopted as standards for a revised system of MK classification for dwarfs.

The relation between $\mathrm{TiO}$ band strength and near-infrared color temperature for dwarfs differs significantly from the giant relation. The coolest dwarfs observed were Wolf 359 and Proxima Centauri; although Wolf 359 is both cooler and less luminous than Proxima, they have nearly identical TiO band strengths.
\end{abstract}

\section{Existing Systems of Classification}

The current state of spectral classification of $M$ dwarfs is unsatisfactory for two reasons. First, the accuracy of one-half a subclass normally achieved for these stars is very low when considered in the light of the physical variables of their atmospheres. The lower main sequence is so steep (about $1.5 \mathrm{mag}$. per spectral subdivision at dM4) that stars that are barely distinguishable spectroscopically on the existing systems (say, stars of types dM4 and dM4.5) differ by more than a factor of two in total energy output. More than $20 \mathrm{yr}$ ago Joy (1947) made this point and emphasized that more accurate types were needed for dwarfs, but until now they have not been provided.

The second problem is that not one but several systems of classification exist for $\mathbf{M}$ dwarfs. For most other kinds of stars it is generally agreed that the MK system should be used, but for M dwarfs the MK system was never really defined. Johnson and Morgan (1953) state that MK types for dwarfs should be expressed on the system of Kuiper (1942), which is essentially the same as the one used earlier by Morgan (1938), on which the latest dwarfs are called M8. In practice, however, MK types that have been assigned to dwarfs (as identified by the Roman numeral $\mathrm{V}$ ) tend to be intermediate to those given by Kuiper (1942) and those given by Joy (1947), on whose system the latest dwarfs are called M6. In particular, Johnson and Morgan adopted type M5V for Barnard's star, which had been called M5 + by Kuiper and M4.5 by Joy.

The various classification systems for $M$ dwarfs are similar in that they all use the absolute TiO band strength as the sole criterion of temperature class. They differ only in that they assign different types to a given band strength. Joy (1947) classified dwarfs on the same scale that was used for giants, namely the Mount Wilson scale, whereas on Kuiper's (1942) system and the MK system the sequence of dwarf types was established completely independently of the giant scale. The scale differences of these systems are well known to those who assign the types, but less well known to other kinds 
of astronomers who have to use them. Not infrequently, one finds types on all three systems in the same catalogues or lists, and if the types are not individually referenced, the uncertainty in their meaning is much greater than the half-subclass accuracy of the original classifications.

\section{Rationale of the Revised System}

The writer is currently reviewing and revising the existing systems of classification for $M$ stars with the aid of photoelectric measurements of TiO band strengths. Traditionally, the temperature classes of $\mathrm{M}$ stars of all luminosities have been based almost entirely upon $\mathrm{TiO}$ strengths, with atomic lines and line ratios serving at most as supplemental criteria. The use of narrow-band photometry increases the accuracy of the TiO measurements, thereby improving the accuracy of the spectral types, without changing the meaning of the types assigned. Thus in the case of the temperature classes of $\mathrm{M}$ stars - and probably only in this case - it is possible to assign MK types (i.e., types based on MK criteria) by means of narrow-band photometry. On the other hand, the MK luminosity classes of $\mathrm{M}$ stars are based upon atomic line ratios and require the higher spectral resolution of classification spectrograms; narrow-band photometry is capable of distinguishing dwarfs, giants, and supergiants of type $M$ in a number of ways, but not by the MK criteria.

For the $\mathrm{M}$ giants, a revised list of MK standards has recently been prepared by Wing and Keenan (1973). The temperature classes, from photometric measures on the 8-color system described below, are given to tenths of subclasses and extend from K4.0-M6.0; the luminosity classes are based upon classification spectrograms, which were also used to remove peculiar stars from the list of standards. A study of the $M$ supergiants, again employing 8-color observations, has recently been completed by White (1971), and spectral types for more than 100 supergiants will be published by White and Wing (1973). In assigning these types, we have used the scale established for giants by Wing and Keenan, rather than the types previously assigned to supergiant standards; the systematic change in the system thereby introduced is smaller than the intrinsic ranges of most supergiants, which vary typically by about one subclass.

In the present paper, 8-color observations are used to define spectral types for $M$ dwarfs, and a table of the revised types of stars suitable for use as standards is presented (see Table II below). The same scale has been used for dwarfs as for giants and supergiants - that is, a given band strength corresponds to a given type regardless of the luminosity class. Of course, a uniform scale of this sort is not necessary for accurate classification, since unknown stars normally are (and will continue to be) classified by direct comparison with standards of the same luminosity class; but it is certainly simpler than having three arbitrary scales for the three main luminosity divisions, coinciding in some intervals of spectral type but diverging in others, as has been the case on the MK system. With the objective measures of the 8-color photometry, the establishment of a uniform scale presents no problem; and since revisions of the types for giant and supergiant standards will soon be published, this seems to be the right 
time to introduce the changes in the types of dwarf standards that are needed to unify the system.

The equality of the strengths of a particular $\mathrm{TiO}$ band in two stars does not, in general, imply that the two stars will also match at all the other TiO bands; weaker or stronger TiO bands may differ if the atmospheric structures are different, and bands in other parts of the spectrum will fail to match if the opacities are different. Thus it is necessary to choose a particular band at which the scales are to be the same, and the $(0,0)$ band of the strong $\gamma$ system, near $7100 \AA$, was chosen when the 8-color system was designed. This band is eminently suitable for photoelectric classification, being easily measurable in $\mathrm{K} 4$ stars (and even in some MK standards of type K3III) and growing to a strength of more than 2 mag. at types beyond M6. Other advantages of this band include its location near the energy maxima of most $M$ stars, its relative freedom from contamination by atomic lines, and its probable immunity to changes resulting from flare activity. A hot thermal continuum superimposed upon the spectrum of a cool dwarf such as Wolf 359, for example, cannot change the type obtained from the $\gamma(0,0)$ band by as much as one-tenth of a subclass unless the $U$ magnitude brightens by more than $2.5 \mathrm{mag}$.

Now that the scale for the spectral classes of $M$ dwarfs has been established through observations of the $\gamma(0,0)$ band, classifications for additional dwarfs on the revised system may of course be obtained by measuring any TiO band with any technique, provided only that similar observations are made for some of the new standard stars.

\section{The Narrow-Band Photometry}

The measurements of TiO band strength used for classifying $\mathrm{M}$ stars have been obtained by photoelectric photometry through interference filters. The complete photometric system of eight filters, described in detail elsewhere (Wing, 1971), serves to measure bands of $\mathrm{CN}$ and VO as well as TiO and to establish the level of the nearinfrared continuum as well as possible in the various kinds of late-type stars. Temperatures are obtained by reducing the measurements to an absolute flux scale - currently the scale of Hayes (1970) is employed - and fitting a blackbody curve through the best continuum points. In the case of $\mathrm{M}$ dwarfs, neither the luminosity-sensitive $\mathrm{CN}$ bands nor the temperature-sensitive VO bands reach detectable strength, so that the 8-color photometry yields only three independent quantities: the infrared magnitude $I(104)$, the color temperature, and the TiO band strength. The absence of $\mathrm{CN}$ and VO bands makes the placement of the continuum particularly secure in this case, while the lack of interstellar reddening is an obvious advantage in discussions of temperatures.

Seven of the eight filters were chosen on the basis of 27-color scanner photometry done at Lick Observatory several years ago (Wing, 1967). The one exception is the filter of shortest wavelength, centered at $7117 \AA$, which measures the $(0,0)$ band of the $\gamma$ system of TiO. This measurement gives the 8-color system a much greater sensitivity to spectral type than the scanner system, which measured only relatively weak bands longward of $7500 \AA$. 


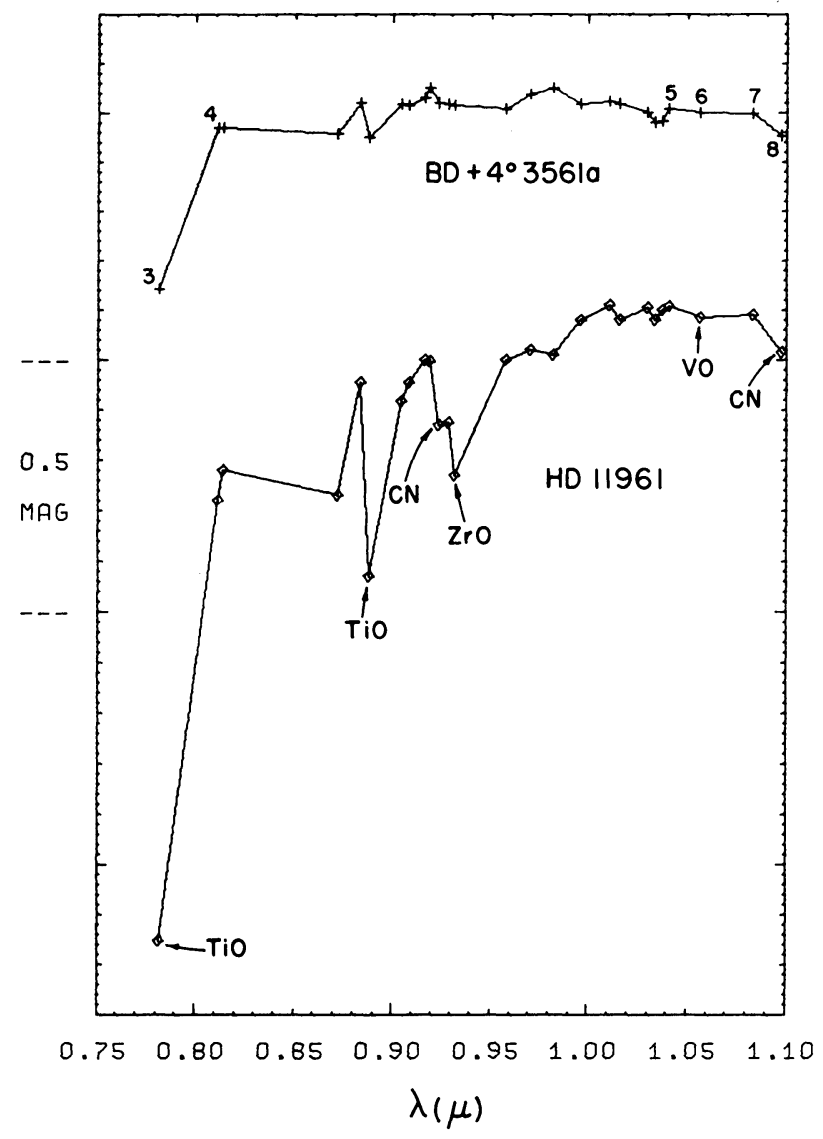

Fig. 1. Scanner spectra of Barnard's star (above) and HD 11961, obtained at Lick Observatory. These stars have previously been classified M5V and M5III, respectively; their revised MK types, reflecting the difference in TiO band strength, are M3.7V and M5.5III. Fluxes per unit wavelength interval, measured at 26 wavelengths with a $30 \AA$ band pass, are plotted on a magnitude scale. The numbers on the upper spectrum indicate the wavelengths corresponding to six of the filters on the 8-color system (see Figure 2).

Figure 1 compares the near-infrared spectra of a dwarf and a giant, observed on the Lick scanner system. The upper star is Barnard's star, BD $+4^{\circ} 3561 a^{*}$, which Johnson and Morgan (1953) used as the definition of MK type M5V, and the lower is HD 11961, classified M5III by Keenan (1963). The large difference in TiO strength illustrates the fact that the MK system uses very different scales for dwarfs and giants, especially for types later than M3. Other major differences include the stronger $\mathrm{CN}$ bands and the redder energy distribution of the giant. The molecules $\mathrm{ZrO}$ and $\mathrm{VO}$ are also weakly present in HD 11961.

In Figure 2, the 8-color observations of three dwarf stars are shown together with

* Dr W. Gliese has kindly pointed out to me that Barnard's star is not BD $+4^{\circ} 3561$, as usually given, but may be called $\mathrm{BD}+4^{\circ} 3561 \mathrm{a}$. 


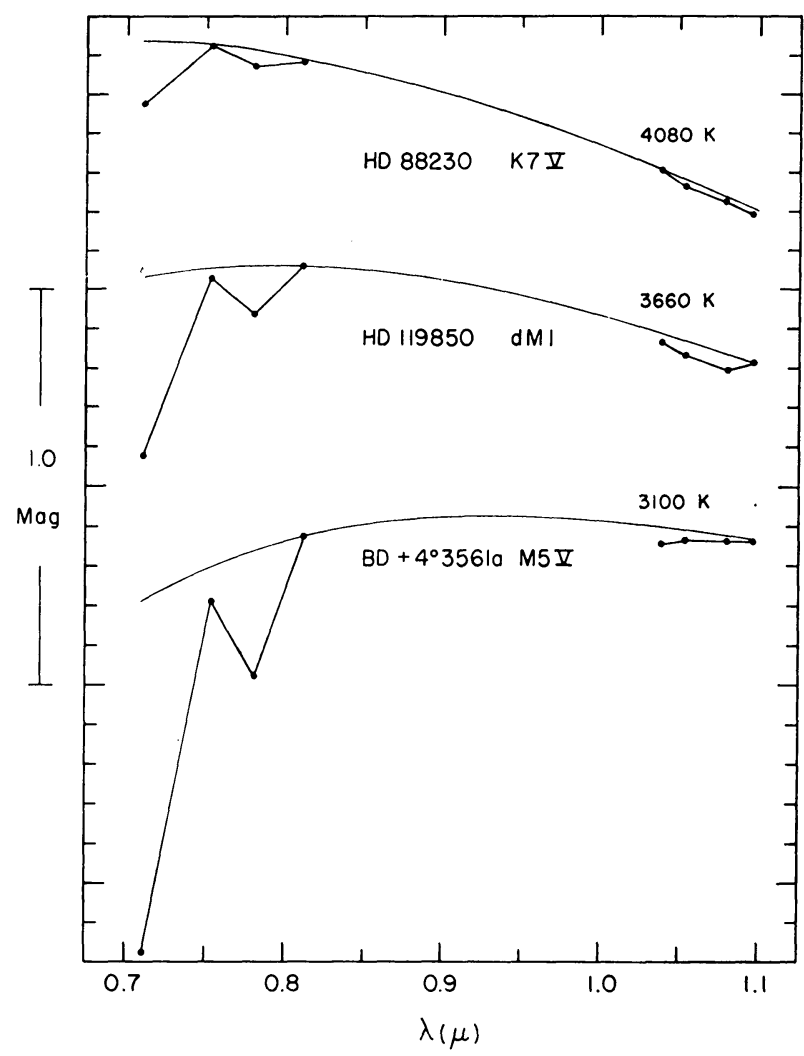

Fig. 2. Spectra on the 8-color system are shown for three late-type dwarfs. The depressions at the first and third filters are caused by TiO. Published spectral types appear after the star names; the revised types, from top to bottom, are $\mathrm{K} 5.1 \mathrm{~V}, \mathrm{M} 1.8 \mathrm{~V}$, and M3.7V. Each spectrum has been fitted with a blackbody curve of the indicated temperature.

their fitted blackbody continua. One of these is again Barnard's star, so that the data on the 8-color and 27-color systems may be compared directly. The numbers 3 through 8 accompanying the spectrum of Barnard's star in Figure 1 indicate the points corresponding most closely to measurements with filters 3 throught 8 on the 8 -color system. Note in particular that the relatively weak TiO band measured by filter 3 is the strongest TiO band included on the scanner program. Filter 2, at $7544 \AA$, corresponds to a point that was added to the 27-color system after the scans of Figure 1 had been obtained.

The index of TiO strength used for classification purposes is defined as the depression at filter 1 with respect to the fitted blackbody continuum, in magnitudes. One advantage of defining the index in this manner is that it is completely uncoupled from the color of the star and hence is independent of reddening (intensity estimates or equivalent width measurements from spectrograms share this characteristic, but most photometric indices do not). It can be seen in Figure 2 that the $\mathrm{TiO}$ index is easily 
measurable in the K7V star with a value of $0.17 \mathrm{mag}$, and that it grows to 0.90 mag. in Barnard's star. Since the uncertainties in the photometry (as judged from the results for standard stars) normally amount to about $\pm 0.015 \mathrm{mag}$. in the magnitudes and $\pm 0.010 \mathrm{mag}$. in the colors, the spectral type interval separating HD 88230 from Barnard's star is about 70 times the error of measurement; thus spectral types may meaningfully be given to tenths of subclasses.

Temperatures of unreddened stars are derived from the 8-color photometry by finding the blackbody curve that passes through one point in each group of four filters and above all the other points (see Figure 2). In most kinds of cool stars, including M dwarfs, it is known from the 27-color photometry (Wing, 1967) that these high points are satisfactorily close to the true continuum. The wavelength baseline is such that an error of $0.01 \mathrm{mag}$. in the photometry at either continuum point introduces an error of about 0.01 in $\theta$, the reciprocal temperature $\left(5040 . \mathrm{T}^{-1}\right)$. At the temperature of Barnard's star, this translates into an uncertainty of $\pm 20 \mathrm{~K}$. Likewise, for each $0.01 \mathrm{mag}$. of error in the absolute calibration between the two continuum points there results a systematic error in all the temperatures amounting to 0.01 in $\theta$. The actual temperatures given in Figure 2 agree well with the work of Johnson (1965); he does not give temperatures for individual stars, but he gives the mean temperatures for types K7V, M1V, and M5V as 4160, 3680, and $3120 \mathrm{~K}$.

The $\mathrm{TiO}$ band strengths are plotted against the reciprocal temperature $\theta$ in Figure 3.

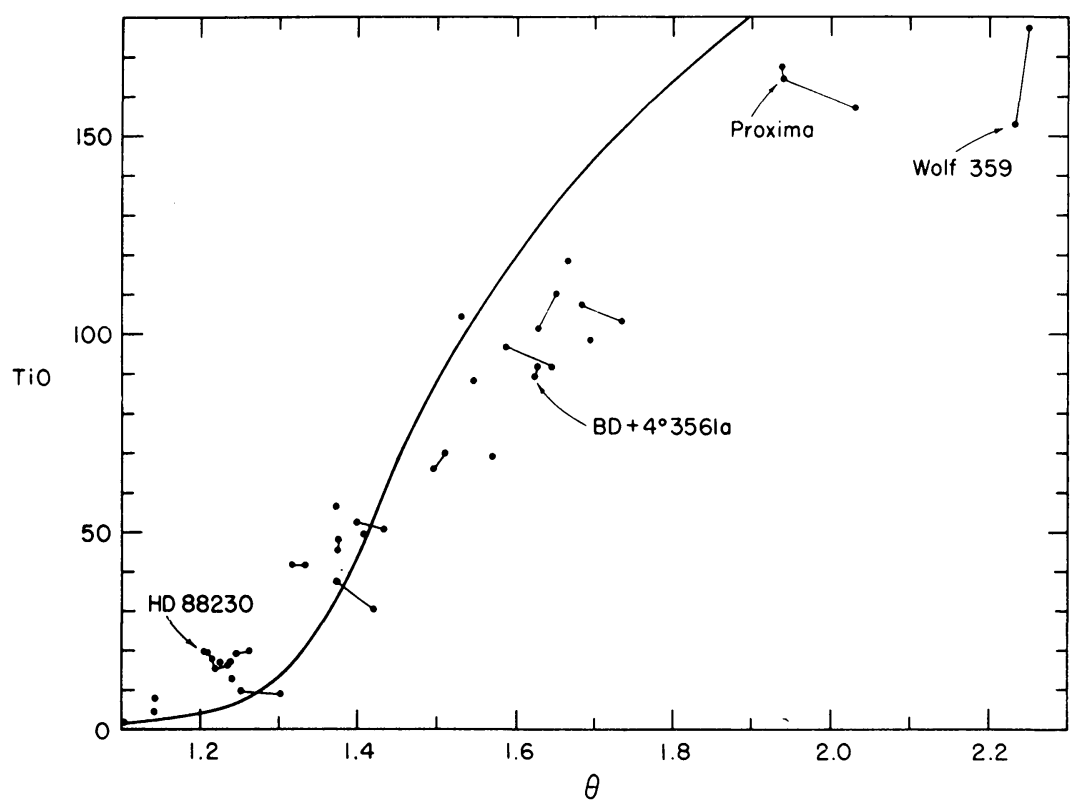

Fig. 3. The TiO index, defined as the depression at filter 1 in units of 0.01 mag., is plotted against the reciprocal temperature $\theta$, obtained from a blackbody fit to the photometry. All observations of dwarfs are plotted, and observations of the same star are connected. The smooth curve is the mean relation for luminosity class III giants. 
All observations of dwarfs later than K4 obtained to date -45 observations of 26 different stars - are plotted, and different observations of the same star are connected by straight lines. The solid curve is the relation for luminosity class III giants, based upon a much larger number of observations. The dwarf relation differs significantly from it, being more nearly linear and also showing more scatter.

Some of the scatter shown by the dwarfs may be due to variability, but the changes shown by stars observed twice are as often perpendicular to the general trend as parallel to it; this suggests that the changes are due mainly to observational scatter, and that the lengths of these line segments may be used to estimate the total observational uncertainty. The probable errors found in this manner are about \pm 0.03 or 0.04 mag., as compared with $\pm 0.01 \mathrm{mag}$. for the colors and indices of standard stars. The difference simply reflects the faintness of the dwarfs, for which the accuracy is limited by photon noise rather than sky quality. The two observations of Wolf 359 , the faintest and reddest dwarf observed, agree well at seven of the eight filters but differ by more than $0.2 \mathrm{mag}$. at filter 1 where the TiO index is obtained; when we consider that in this case we are trying to measure the deepest part of the strongest absorption band in a star of visual magnitude 13.6, the scatter at filter 1 is understandable. This is the worst case, and even here the uncertainties in the resulting spectral types are acceptably small: the two individual classifications of Wolf 359 , on the scale discussed below, are M5.3 and M5.9.

An effort has been made to identify and observe dwarfs that are as cool and as late in type as possible, and several stars previously classified dM5 or later are among those plotted in Figure 3. Most of these stars fall quite close to Barnard's star in the middle of the diagram, while two stars - Proxima Centauri and Wolf 359 - stand out as having much stronger bands and redder colors than the others. Although a few stars are known to exist which have even fainter absolute magnitudes than Wolf 359 (for example, the companion to $\mathrm{BD}+4^{\circ} 4048$, which has not yet been observed on this program) there is at present no evidence for the occurrence of stronger TiO bands in dwarfs than those shown by Proxima and Wolf 359. The upper border of Figure 3 corresponds to type M6.0 on the scale used here.

The supposition that the color difference between Proxima and Wolf 359 represents a real difference in temperature can be supported by three arguments: (1) an infrared spectrogram of Wolf 359 taken by Wing and Ford (1969) shows no molecular absorption that could seriously depress the shortward continuum point; (2) the derived temperature for Wolf $359,2250 \mathrm{~K}$, agrees well with the value $2230 \mathrm{~K}$ obtained from scanner measurements made at the Lick 120-in. telescope by Spinrad and Wing, who used a different shortward continuum point (Wing, 1967); and (3) Wolf 359 is 1.7 mag. fainter that Proxima in $M_{V}$ and hence would be expected to be substantially cooler. If this temperature difference is accepted, then the equality of the band strengths strongly suggests that an upper limit exists to the $\mathrm{TiO}$ band strength attainable in dwarfs. It will be important to determine whether a continuous sequence of star exists between Proxima and Wolf 359, or whether the latter is really a lone wolf.

The differences between the mean relations for dwarfs and giants in Figure 3 can 
probably be accounted for qualitatively in the following manner. In the early $\mathbf{M}$ stars, where only a small fraction of the titanium atoms is in molecular form, the dwarfs have stronger bands than giants of the same temperature as a direct result of their higher gas pressures and molecular concentrations. At the later types, however, when molecule formation is considerable in both dwarfs and giants, the band strength may be governed mainly by the opacity in the continuum; if so, we would infer from Figure 3 that, with decreasing temperature, the opacity either increases in the dwarfs or decreases in the giants, so that the mean relations for the two luminosity classes cross each other. If there exists an upper limit to the TiO band strength in dwarfs, it could be imposed either by increasing opacity or by the formation of titanium dioxide, which should be much more important in dwarfs than in giants.

The observations presented here were obtained with two telescopes: the Perkins 72-in. telescope of the Ohio State and Ohio Weslyan Universities located near Flagstaff, Arizona, and the 60-in. telescope of the Cerro Tololo Inter-American Observatory in Chile. Many of the stars lie in the equatorial region, and several have been observed from both hemispheres.

\section{The Spectral Types}

The reasons for deciding to classify dwarfs on the scale established for giants by Wing and Keenan (1973) have already been stated. Thus, types for dwarfs can be obtained simply by entering the $\mathrm{TiO}$ index, as measured on the 8-color system, into the calibration already derived. Two points, however, need to be discussed.

The first is that type K7, which has been used on the MK system for dwarfs but not for giants, will no longer exist for any luminosity class on the new unified system. Type M0.0 follows immediately after type K5.9, and the types K4.0, K5.0, M0.0, and M1.0 have been defined to represent equal intervals in TiO band strength (and hence nearly equal intervals in the inverse temperature $\theta$ ).

The second point is that absorption by the $\mathrm{CN}$ molecule is nearly continuous throughout the red and infrared, and in particular the region of filter 1 contains fairly strong $\mathrm{CN}$ absorption. In order to obtain spectral types that are independent of the $\mathrm{CN}$ strength and that are truly on the same scale for all luminosity classes, corrections must be applied for the contribution of $\mathrm{CN}$ to the depression of filter 1. Fortunately, the appropriate correction for each star can be derived from the 8-color photometry alone (see White, 1971), since filters 4 and 8 provide explicit $C N$ measurements. Since Wing and Keenan (1973) used $\mathrm{CN}$-corrected TiO indices in deriving the calibration for giants, no further corrections need to be applied here, because the effects of $\mathrm{CN}$ are negligible in $\mathbf{K}$ and $\mathbf{M}$ dwarfs. If the giants had not been corrected, however, $\mathrm{a}$ systematic error of about half a subclass between dwarfs and giants would have been introduced.

The accuracy of the spectral types depends upon the photometric accuracy (already discussed) and the width of the spectral type intervals in terms of the TiO index. In the interval K4.0-M1.0, as defined by Wing and Keenan (1973), the CN-corrected TiO 
index increases by precisely $0.01 \mathrm{mag}$. per spectral type interval of 0.1 subclass, so that tenths of subdivisions may meaningfully be used for the means of repeated observations of non-variable stars or for single observations of bright stars made on good nights. With advancing type, the relation between band strength and spectral type becomes steeper, so that for example each 0.1 subclass has a width of 0.04 mag. in the range M4.0-M6.0. In progressing down the main sequence, the increasing width of these intervals tends to compensate for the fainter apparent magnitudes with which we are forced to contend.

The spectral types for dwarfs obtained to date are given in the last column of Tables I and II, under the heading 'revised MK type'. It is hoped that these types will not only be considered to be MK types but will be adopted as the definition of the MK scale for dwarfs. The new types are compared with types given by Kuiper (1942) and Joy (1947), and with those found in the literature expressed in the MK notation.

Table I gives the mean types for stars observed more than once ( $n$ is the number of observations), and it is these stars that are recommended for use as standards of spectral type. The list is too short to give a really adequate distribution in both right ascension and spectral type, but most of the stars are observable from both hemispheres and additional standards will be made available in the near furture. Some of these stars undergo flare activity, but none is known definitely to be variable in spectral type or in the infrared continuum. The degree of agreement of the different observations of these stars can be seen by inspection of Figure 3. From the considerations discussed earlier, the mean types in Table I are judged to have probable errors of \pm 0.1 subclass and maximum errors of \pm 0.2 subclass. In Table II are given the types obtained for stars observed only once; their uncertainties depend somewhat upon the circumstances of the particular observation, but estimates for typical cases indicate probable errors of \pm 0.2 subclass and maximum errors of \pm 0.3 subclass.

TABLE I

Spectral types for standard stars

\begin{tabular}{|c|c|c|c|c|c|c|c|}
\hline Star & $\alpha(1900)$ & $\delta(1900)$ & $\begin{array}{l}\text { Kuiper } \\
\text { (1942) }\end{array}$ & $\begin{array}{l}\text { Joy } \\
(1947)\end{array}$ & $\begin{array}{l}\text { Published } \\
\text { MK type }\end{array}$ & $n$ & $\begin{array}{l}\text { Revised } \\
\text { MK type }\end{array}$ \\
\hline HR 753 B & $2^{\mathrm{h}} 30^{\mathrm{m}} 6$ & $+6^{\circ} 25^{\prime}$ & M6 & - & - & 2 & M4.1V \\
\hline HD 33793 & 507.7 & -4459 & Mo & - & MOV & 2 & K4.1V \\
\hline $\mathrm{BD}-12^{\circ} 2918$ & 926.4 & $-13 \quad 03$ & M4 & - & - & 2 & M2.9V \\
\hline HD 88230 & 1005.3 & +4958 & $\mathrm{~K} 8$ & - & $\mathrm{K} 7 \mathrm{~V}$ & 6 & $\mathrm{~K} 5.1 \mathrm{~V}$ \\
\hline Wolf 359 & 1051.6 & +737 & M8 & dM6e & - & 2 & M5.6:V \\
\hline Ross 128 & 1142.6 & +123 & M5+ & dM5 & - & 2 & M4.1V \\
\hline HD 111631 & 1245.6 & -013 & K8 & - & $\mathrm{M} 0.5 \mathrm{~V}$ & 2 & $\mathrm{~K} 5.3 \mathrm{~V}$ \\
\hline $\mathrm{BD}+11^{\circ} 2576$ & 1324.9 & +1055 & M1 & dM1 & M1V & 2 & $\mathrm{M} 0.8 \mathrm{~V}$ \\
\hline HD 119850 & 1340.7 & +1526 & $\mathbf{M} 1+$ & - & M4V & 2 & M1.8V \\
\hline Proxima Cen & 1422.8 & $-62 \quad 15$ & - & - & - & 3 & M5.6V \\
\hline $\mathrm{BD}+4^{\circ} 3561 \mathrm{a}$ & 1752.9 & +425 & M5+ & sdM4.5 & M5V & 2 & M3.7V \\
\hline $\mathrm{BD}-15^{\circ} 6290$ & 2247.9 & -1447 & M5 & $\mathrm{dM} 4.5$ & - & 2 & M3.8V \\
\hline HD 217987 & 2259.4 & -3626 & M0 & - & M2V & 2 & $\mathrm{M} 1.5 \mathrm{~V}$ \\
\hline HD 225213 & 2359.5 & -3751 & M3 & - & M4V & 2 & M2.1V \\
\hline
\end{tabular}


TABLE II

Spectral types for additional stars

\begin{tabular}{|c|c|c|c|c|c|c|}
\hline Star & $\alpha(1900)$ & $\delta(1900)$ & $\begin{array}{l}\text { Kuiper } \\
\text { (1942) }\end{array}$ & $\begin{array}{l}\text { Joy } \\
(1947)\end{array}$ & $\begin{array}{l}\text { Published } \\
\text { MK type }\end{array}$ & $\begin{array}{l}\text { Revised } \\
\text { MK type }\end{array}$ \\
\hline $\mathrm{BD}+5^{\circ} 1668$ & $7^{\mathrm{h}} 22^{\mathrm{m}} 0$ & $+5^{\circ} 31^{\prime}$ & M4 + & $\mathrm{dM} 4$ & - & M4.1V \\
\hline Wolf 358 & 1045.8 & +722 & M5 & dM5 & - & $\mathrm{M} 4.0 \mathrm{~V}$ \\
\hline $\mathrm{BD}-11^{\circ} 3759$ & 1428.9 & -1206 & M4 & - & - & $\mathrm{M} 4.5 \mathrm{~V}$ \\
\hline HR 5568 A & 1451.6 & -2058 & K4 & - & K5V & $\mathrm{K} 3.5 \mathrm{~V}$ \\
\hline B & & & M0 + & - & M2V & M2.0V \\
\hline BD $-7^{\circ} 4003$ & 1514.2 & -721 & M4 & - & _- & M3.0V \\
\hline BD $-12^{\circ} 4523$ & 1624.7 & -1225 & M4 & $\mathrm{dM} 4.5$ & - & M3.6V \\
\hline HD 154363 & 1659.8 & -454 & - & - & K5V & $\mathrm{K} 4.2 \mathrm{~V}$ \\
\hline BD $-4^{\circ} 4226$ & 1700.0 & -455 & - & - & M3.5V & $\mathrm{M} 2.4 \mathrm{~V}$ \\
\hline HD 157881 & 1720.8 & +214 & K7 & - & K7V & $\mathrm{K} 5.0 \mathrm{~V}$ \\
\hline 61 Cyg A & 2102.4 & +3815 & $\mathrm{~K} 3$ & - & K5V & $\mathrm{K} 3.5 \mathrm{~V}$ \\
\hline B & & & $\mathrm{K} 5$ & - & K7V & $\mathrm{K} 4.7 \mathrm{~V}$ \\
\hline
\end{tabular}

\section{Plans for Further Work}

The observations presented here represent approximately the first $20 \%$ of a program intended to furnish two or more observations of each of about $100 \mathrm{M}$ dwarfs. The observing list includes the several brightest single stars of each spectral type, with emphasis on the latest types. The 8-color photometry will provide color temperatures and $I(104)$ magnitudes in the infrared continuum, as well as spectral types. Discussion of the following topics is planned, but will be deferred until the observational data are complete:

(1) By combining the 8-color photometry with trigonometric parallaxes, a colormagnitude diagram involving the absolute $I(104)$ magnitude and the reciprocal temperature $\theta$ will be constructed in order to discuss once again such topics as the width and slope of the lower main sequence, and the existence of subdwarfs.

(2) New values for the mean colors, effective temperatures, and bolometric corrections as a function of spectral type will be derived on the basis of the wideband photometry published by Johnson (1965), simply by regrouping the stars he observed according to the new and more self-consistent spectral types.

(3) Algebraic relations will be derived whereby the bolometric magnitude and effective temperature are expressed as functions of the $I(104)$ magnitude, the reciprocal color temperature $\theta$, and the spectral type, all of which are given directly by the 8 -color photometry.

\section{Acknowledgements}

Support from the National Science Foundation is gratefully acknowledged. I would like to thank the Director and staff of the Cerro Tololo Inter-American Observatory for their hospitality and for observing time on the 60-in. telescope, with which most of the data discussed here were obtained. 


\section{References}

Hayes, D. S.: 1970, Astrophys. J. 159, 165.

Johnson, H. L.: 1965, Astrophys. J. 141, 170.

Johnson, H. L. and Morgan, W. W.: 1953, Astrophys. J. 117, 313.

Joy, A. H.: 1947, Astrophys. J. 105, 96.

Keenan, P. C.: 1963, in K. Aa. Strand (ed.), Basic Astronomical Data, University of Chicago Press, Chicago, p. 78.

Kuiper, G. P.: 1942, Astrophys. J. 95, 201.

Morgan, W. W.: 1938, Astrophys. J. 87, 589.

White, N. M.: 1971, Dissertation, The Ohio State University.

White, N. M. and Wing, R. F.: 1973, in preparation.

Wing. R. F.: 1967, Dissertation, University of California, Berkeley.

Wing, R. F.: 1971, in G. W. Lockwood and H. M. Dyck (eds.), Proc. of the Conference on Late-Type Stars, Kitt Peak National Observatory Contribution No. 554, p. 145.

Wing, R. F. and Ford, W. K., Jr.: 1969, Publ. Astron. Soc. Pacific 81, 527.

Wing, R. F. and Keenan, P. C.: 1973, in preparation. 